\title{
Anomalous unidirectional excitation of high-k hyperbolic modes using all-electric metasources
}

\author{
Zhiwei Guo $\oplus_{,}^{\text {a,b, },, \dagger}$ Yang Long, ${ }^{\mathrm{a}, \mathrm{c}, *, \dagger}$ Haitao Jiang, ${ }^{\mathrm{a}, \mathrm{b}}$ Jie Ren, ${ }^{\mathrm{a}, \mathrm{c}}$ and Hong Chen ${ }^{\mathrm{a}, \mathrm{b}, \mathrm{c}}$ \\ ${ }^{a}$ Tongji University, School of Physics Science and Engineering, Shanghai, China \\ ${ }^{\mathrm{b}}$ Tongji University, MOE Key Laboratory of Advanced Micro-Structure Materials, Shanghai, China \\ ${ }^{c}$ Tongji University, Center for Phononics and Thermal Energy Science, China-EU Joint Lab on Nanophononics, \\ Shanghai Key Laboratory of Special Artificial Microstructure Materials and Technology, Shanghai, China
}

\begin{abstract}
The unidirectional excitation of near-field optical modes is a fundamental prerequisite for many photonic applications, such as wireless power transfer and information communications. We experimentally construct all-electric Huygens and spin metasources and demonstrate anomalous unidirectional excitation of high- $k$ hyperbolic modes in two types of hyperbolic metasurfaces. We use a Huygens metasource to study the unidirectional excitation of hyperbolic bulk modes in a planar hyperbolic metamaterial (HMM). Specifically, unidirectional excitation is the same as that in free space in the vertical direction, but opposite to that in free space in the horizontal direction. This anomalous unidirectional excitation is determined by the anisotropic HMM dispersion. In addition, we use a spin metasource to observe the anomalous photonic spin Hall effect in a planar hyperbolic waveguide. For a near-field source with a specific spin, the guide mode with a fixed directional wave vector is excited due to spin-momentum locking. Because the directions of momentum and energy flows in the HMM waveguide are opposite, the unidirectional excitation of hyperbolic guided modes is reversed. Our results not only uncover the sophisticated electromagnetic functionalities of metasources in the near-field but may also provide novel opportunities for the development of integrated optical devices.
\end{abstract}

Keywords: hyperbolic metasurfaces; topological transition; guided modes; circuit-based metamaterials; metasources.

Received Mar. 10, 2021; revised manuscript received May 12, 2021; accepted for publication May 20, 2021; published online Jun. 9, 2021.

(C) The Authors. Published by SPIE and CLP under a Creative Commons Attribution 4.0 Unported License. Distribution or reproduction of this work in whole or in part requires full attribution of the original publication, including its DOI.

[DOI: 10.1117/1.AP.3.3.036001]

\section{Introduction}

Hyperbolic metamaterials (HMMs), an important class of artificial anisotropic material with hyperbolic isofrequency contours (IFCs), have recently attracted significant attention due to their unique ability to control interactions between light and matter. ${ }^{1-4}$ Tuning the hyperbolic dispersion shape allows light propagation in HMMs to be flexibly controlled to produce all-angle negative refraction, ${ }^{5-8}$ collimation, ${ }^{9,10}$ beam splitting, ${ }^{11,12}$ and robust transmission. ${ }^{13,14}$ Tuning the topological transition of the dispersion from a closed IFC to an open hyperbolic IFC significantly enhances the optical density of states (DOS). This has important consequences for the strong enhancement of spontaneous

*Address all correspondence to Zhiwei Guo, 2014guozhiwei@tongji.edu.cn; Yang Long, longyang_123@yeah.net

†These authors contributed equally to this work. emission. ${ }^{15,16}$ In addition, HMMs can convert evanescent waves into propagating waves with large wave vectors. This property has enabled the demonstration of superresolution imaging that overcomes the diffraction limit ${ }^{17,18}$ and long-range dipole-dipole interactions beyond the near-field coupling limitation. ${ }^{19-21}$ Interestingly, under near-field excitation, electromagnetic (EM) waves in HMMs propagate along fixed channels at the subwavelength scale. This is similar to crossing waveguides. ${ }^{22}$ These extraordinary guided modes with strong spatial localization correspond to high- $k$ modes with large DOSs in HMMs. In 2014, Kapitanova et $\mathrm{al}^{23}$ experimentally demonstrated the photonic spin Hall effect (PSHE) based on spin-orbit coupling of extraordinary guided modes in HMMs. This optical spin-orbit locking phenomenon comes from the transverse spin property of evanescent waves. ${ }^{24-31}$ Unlike with the PSHE in surface plasmon polaritons at a metal-dielectric interface, ${ }^{32-34}$ the directional excitation of spin dipoles in HMMs occurs inside the bulk of the structure. This 
greatly broadens the near-field coupling control scope. ${ }^{35}$ Although the spin dipole can achieve unidirectional excitation based on spin-orbit coupling, switchable and flexibly controlled unidirectional excitation in HMMs is a highly concerning topic. ${ }^{36}$ Diversified unidirectional emission may facilitate new wireless power transfer ${ }^{37}$ and information communication ${ }^{38}$ applications.

An alternative way to achieve unidirectional emission is the Huygens source. As a coherent dipole with orthogonal electrical and magnetic dipole resonances, a Huygens source can produce directional radiation in the far field when the Kerker condition is satisfied..$^{39}$ Although the Huygens source was originally introduced as a fictitious entity, some effective approaches to implementing it using polarizable subwavelength particles that sustain both electric and magnetic dipolar resonances have been proposed. ${ }^{40}$ Thus far, the Huygens source has attracted extensive attention for production of unidirectional far-field antennas. ${ }^{41,42}$ Based on this far-field property, efficient Huygens metasurfaces with arbitrary EM wave fronts have recently been proposed for all-dielectric ${ }^{43-46}$ and actively controlled systems. ${ }^{47}$ Specifically, Picardi et al.$^{48}$ theoretically revealed the near-field directionality of a Huygens dipole. The switchable excitation directions of the Huygens dipole are different from those of spin dipoles because of obvious differences in symmetry characteristics. ${ }^{49}$ These remarkable findings show that the near-field properties of a Huygens dipole can provide new physical insights into various unidirectional near-field couplings. ${ }^{48-50}$

In this paper, we use two-dimensional (2D) transmission lines (TLs) with lumped elements to design and fabricate circuit-based HMMs and hyperbolic waveguides. Then, we experimentally construct all-electric Huygens and spin metasources and demonstrate anomalous unidirectional excitation of high- $k$ hyperbolic modes. In particular, we experimentally observe the anomalous unidirectional excitation of hyperbolic bulk modes in the horizontal direction using a Huygens metasource. Moreover, we study the anomalous unidirectional excitation of hyperbolic guided modes using the spin metasource. Our results not only clearly show the interesting near-field unidirectional emissions of the Huygens metasource in HMMs and spin metasources in hyperbolic waveguides, but also provide a flexible platform for the construction of more complex composite metasources. Related designs can be extended to the fields of natural 2D materials $^{51,52}$ and acoustics systems. ${ }^{31,53,54}$

\section{Unidirectional Excitation of Hyperbolic Bulk Modes Using a Huygens Metasource}

The unidirectional excitation of magnetic metamaterials for transverse-electric-polarized (TE-polarized) waves has recently attracted extensive attention. ${ }^{55-58}$ In this section, we present near-field unidirectional excitation of a Huygens metasource in planar magnetic HMMs, ${ }^{59,60}$ which may be easy to integrate, exhibit small losses, and facilitate new applications such as energy transfer and switching. The emission properties of EM waves in media depend on their dispersion in wave vector space, which is characterized by their IFCs. Figure 1 shows examples of closed ellipsoid and open hyperboloid IFCs. Upon comparing Fig. 1(a) with Fig. 1(b), it is apparent that the HMM, which has an open IFC, has a diverging shell volume. This implies that the ideal HMM can support an infinite optical DOS. ${ }^{15,16}$ Because of the special HMM dispersion, the propagation direction of a wave in an HMM is different from that in a normal anisotropic material with a closed IFC. Based on the boundary conditions and the causality law, when a wave with a positive wave vector $\left(k_{x}>0, k_{y}>0\right.$, and $\left.k_{z}>0\right)$ is incident on a normal material, the energy flows in all directions are positive [Fig. 1(a)]. However, when a wave with a positive wave vector $\left(k_{x}>0\right.$, $k_{y}>0$, and $k_{z}>0$ ) is incident on an HMM, the energy flow in the $z$ - $(x$ - or $y$-) direction is positive (negative), as shown in Fig. 1(b). We reveal that hyperbolic bulk modes can be used to achieve anomalous unidirectional excitations during near-field excitations of an all-electric Huygens metasource.

We calculate radiation patterns for simple point dipoles in air and HMM $\left(\mu_{z}=-1.47, \mu_{x}=\mu_{y}=1\right.$, and $\left.\varepsilon=3.57\right)$ using the finite-element method module of COMSOL Multiphysics. These

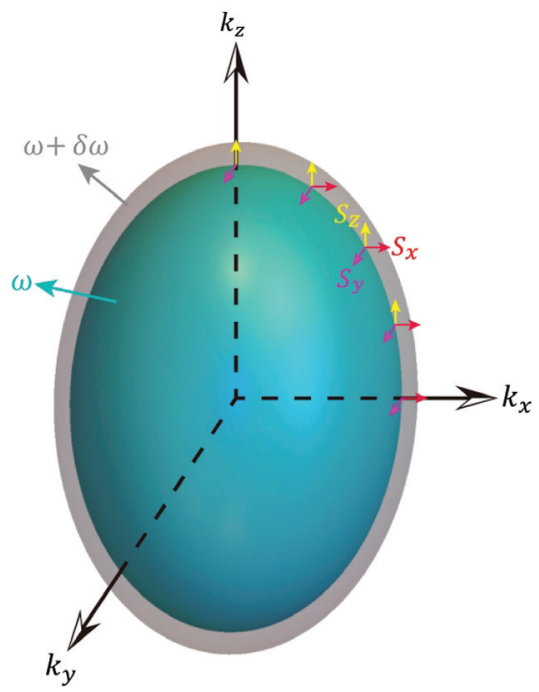

(a)

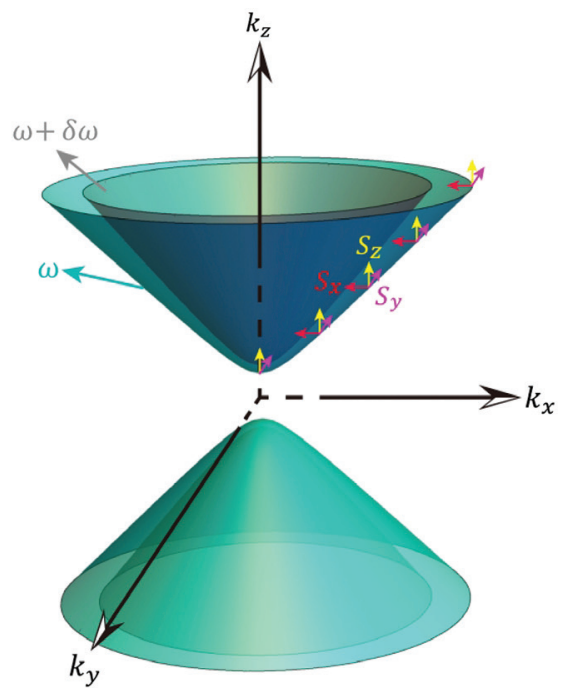

(b)

Fig. 1 Various 3D IFCs for (a) a closed ellipsoid and (b) an open hyperboloid when the frequency increases from $\omega$ to $\omega+\delta \omega$. The energy flows in the $x, y$, and $z$ directions are marked using red, pink, and yellow arrows, respectively. 


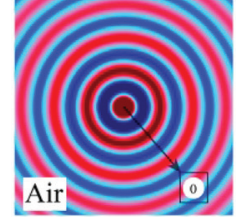

(a)
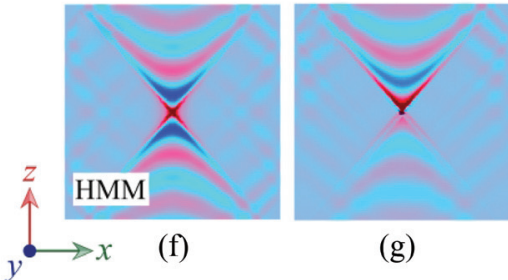

(g)

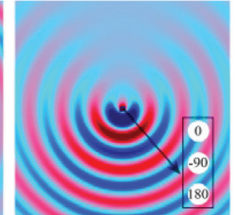

(c)

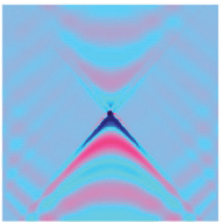

(h)

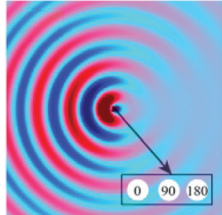

(d)

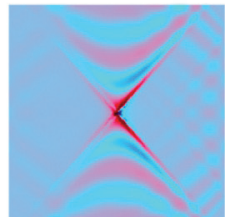

(i)

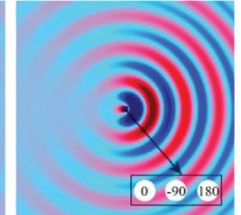

(e)

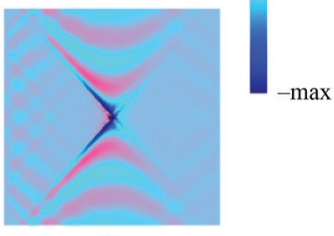

(j)

Fig. 2 (a) Radiation patterns for a simple point dipole in air, where the EM waves can propagate along all directions. (b)-(e) Unidirectional propagation from the Huygens metasource in air. (f) Radiation patterns for a simple point dipole in HMM, where the EM waves propagate mainly along the four channels with high- $k$ modes. Panels $(\mathrm{g})-(\mathrm{j})$ are similar to $(\mathrm{b})-(\mathrm{e})$ but for unidirectional propagation of the Huygens metasources in an HMM.

are shown in Figs. 2(a) and 2(f), respectively. A comparison of Figs. 2(a) and 2(f) clearly shows that light propagates along all inplane directions in air but only along a certain range of angles in the HMM. Specifically, the field is much stronger at hyperbolic asymptotes than elsewhere because of the larger optical DOS. ${ }^{23}$ Then, we numerically study near-field unidirectional excitation by all-electric Huygens metasources in air and HMMs, as shown in Figs. 2(b)-2(e) and Figs. 2(g)-2(j), respectively. The Huygens metasource is composed of three dipole sources separated by small spacings $(d \ll \lambda)$. These discrete dipoles have the same intensity but different phases along the horizontal and vertical directions. ${ }^{49}$ Since the all-electric Huygens metasource is composed of three electric dipoles arranged successively with phases of $0 \mathrm{deg}, 90 \mathrm{deg}$, and $180 \mathrm{deg}(0 \mathrm{deg},-90 \mathrm{deg}$, and $180 \mathrm{deg})$ along the vertical direction ( $z$ direction), the EM waves cause upward (downward) unidirectional excitation in air, as shown in Fig. 2(b) [Fig. 2(c)]. The results are similar for the HMMs in Figs. 2(g) and 2(h). However, anomalous unidirectional excitation occurs in HMMs when the Huygens metasource is constructed along the horizontal direction ( $x$ direction). The direction of the leftward (rightward) unidirectional excitation of Huygens metasources at $0 \mathrm{deg}, 90 \mathrm{deg}$, and $180 \mathrm{deg}(0 \mathrm{deg},-90 \mathrm{deg}$, and $180 \mathrm{deg}$ ) in air [Figs. 2(d) and 2(e)] changes to rightward (leftward) unidirectional excitation in an HMM [Figs. 2(i) and 2(j)].

To understand anomalous unidirectional phenomena in the HMM, we consider the HMM with $\mu_{x}=\mu_{y}=\mu>0, \mu_{z}<0$, and $\varepsilon>0$. The dispersion in the $x o z$ 2D plane is $\frac{k_{x}^{2}}{\mu_{z}}+\frac{k_{z}^{2}}{\mu}=\varepsilon\left(\frac{\omega}{c}\right)^{2}$, with the bulk mode as $E_{y}=A, E_{x}=E_{z}=0, H_{x}=-A \frac{c}{\omega} \frac{k_{z}}{\mu}$, $H_{y}=0$, and $H_{z}=A \frac{c}{\omega} \frac{k_{x}}{\mu_{z}}$, where $A$ is a constant, $c$ is the speed of light in vacuum, and $k_{x}$ and $k_{z}$ are the $x$ and $z$ components of the wave vector, respectively. According to the electric field of this bulk mode, the eigenfunction for the frequency $\omega_{k}$ can be written as $\boldsymbol{u}_{\boldsymbol{k}}=(0,1,0) e^{i\left(k_{x} x+k_{z} z\right)}, \boldsymbol{k}=\left(k_{x}, 0, k_{z}\right)$, and the Green function will be ${ }^{61}$

$G\left(r, r^{\prime}, \omega\right)=\sum_{k} c^{2} \frac{\boldsymbol{u}_{\boldsymbol{k}}^{*}\left(\boldsymbol{r}^{\prime}, \omega_{k}\right) \boldsymbol{u}_{\boldsymbol{k}}\left(\boldsymbol{r}, \omega_{k}\right)}{\omega_{k}^{2}-\omega^{2}}$.
The Huygens metasource can be composed of three phasedelayed electric dipoles separated by a distance $d$. For example, the horizontally placed Huygens metasource can be expressed as $\quad p(\boldsymbol{r}, t)=p_{0} \boldsymbol{e}_{y}[\delta(x) \pm i \delta(x-d) \mp i \delta(x+d)] e^{-i \omega t}=$ $p(\boldsymbol{r}, \omega) e^{-i \omega t}$. The excited electric field will be $\boldsymbol{E}(\boldsymbol{r}, \omega)=$ $\int_{-\infty}^{\infty} \omega^{2} \mu_{0} G\left(\boldsymbol{r}^{\prime}, \boldsymbol{r}, \omega\right) \boldsymbol{p}\left(\boldsymbol{r}^{\prime}, \omega\right) \mathrm{d} \boldsymbol{r}^{\prime}$, and the component after the Fourier transformation becomes

$$
\begin{aligned}
E_{y}\left(k_{x}, k_{y}, \omega_{k}\right) & =\frac{\mu_{0} c^{2} \omega^{2}}{\omega_{k}^{2}-\omega^{2}} p_{0} F_{k} \\
& =\frac{\mu_{0} c^{2} \omega^{2}}{\omega_{k}^{2}-\omega^{2}} p_{0}\left(1 \pm i e^{i k_{x} d} \mp i e^{-i k_{x} d}\right),
\end{aligned}
$$

where $F_{k}=1 \pm i e^{i k_{x} d} \mp i e^{-i k_{x} d}$ is the excitation factor raised by the source array. One can determine that the time-averaged Poynting vector for the bulk mode $\boldsymbol{u}_{\boldsymbol{k}}$ is $\boldsymbol{S}=\frac{1}{2} \operatorname{Re}\left[\boldsymbol{E}^{*} \times \boldsymbol{H}\right]=$ $\frac{c|A|^{2}}{2 \omega}\left(\frac{k_{x}}{\mu_{z}}, 0, \frac{k_{z}}{\mu}\right)^{\mathrm{T}}$. Using the propagation angle $\theta=\arg \left[S_{x}+i S_{z}\right]=$ $\arg \left[\frac{k_{x}}{\mu_{z}}+i \frac{k_{z}}{\mu}\right]$ of the excited electric field, we can obtain the relation between the excitation factor $F_{k}$ and the propagation angle $\theta$, as shown in Fig. 3. Comparing the relation between $F_{k}$ and $\theta$ in air (the orange line in Fig. 3), we can see that the Huygens metasource placed along the vertical direction excites the HMM mode with the same directionality as in air [Figs. 3(a) and 3(b)]. However, the horizontally placed metasource produces the opposite directionality [Figs. 3(c) and 3(d)]. The main physical reason behind the anomalous excitation phenomena for the horizontally placed Huygens metasource is due to the special HMM dispersion, as shown in Fig. 1(b). The anomalous excitation phenomena appear because of the directional mismatch between the wave vector $\boldsymbol{k}=\left(k_{x}, 0, k_{z}\right)^{\mathrm{T}}$ and the time-averaged energy flow $S=\frac{c|A|^{2}}{2 \omega}\left(\frac{k_{x}}{\mu_{z}}, 0, \frac{k_{z}}{\mu}\right)^{\mathrm{T}}$ for the HMM $\left(\mu_{z}<0, \mu>0\right)$. Obviously, the extraordinary guide mode present in HMMs makes them a good research platform for the study of abundant unidirectional transmission.

Based on 2D TLs with lumped elements in the microwave regime, we construct circuit-based magnetic HMMs and 


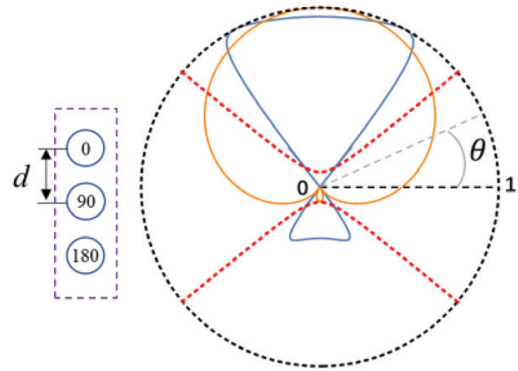

(a)

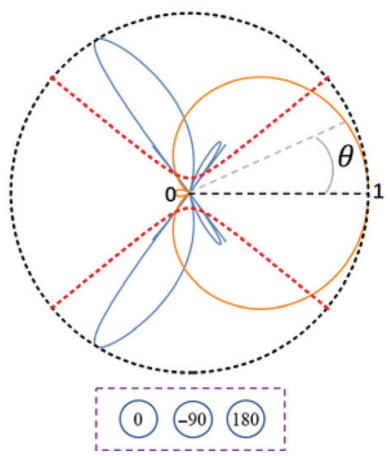

(c)

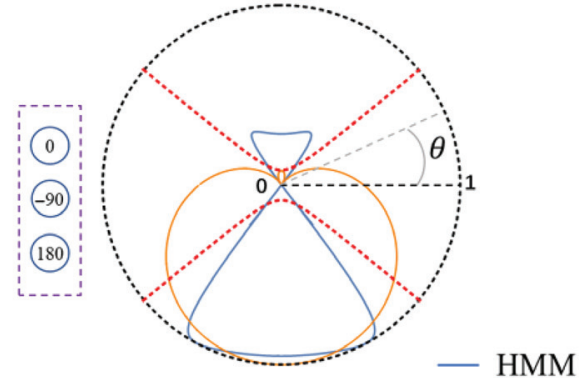

(b)

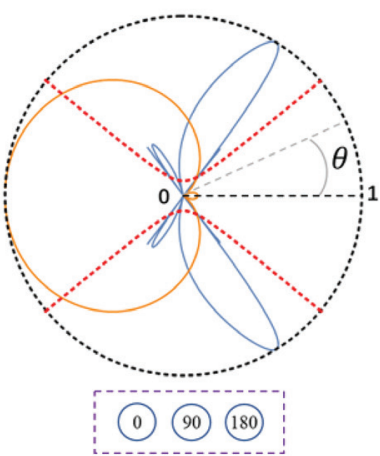

(d)

Fig. 3 The $\left|F_{k}\right|$ of the Huygens metasources as functions of the propagation direction $\theta$ in different settings. The Huygens metasources are shown in the purple boxes with numbers that indicate the phase delay (degree unit) of each excitation source. The $\left|F_{k}\right|$ functions (normalized by their maximum values) in the HMM and air are denoted by blue and orange lines, respectively. The dashed red and black lines indicate the HMM dispersion $\omega\left(k_{x}, k_{z}\right)$ and the maximum value of $\left|F_{\boldsymbol{k}}\right|$. Here, $d=0.1 \lambda$, where $\lambda$ is the wavelength in vacuum, and $p_{0}=1$.

experimentally demonstrate anomalous unidirectional transmissions from Huygens metasources in HMMs. A schematic of the effective HMM is shown in Fig. 4(a). Our structure is constructed on a commercially printed F4B circuit board (relative permittivity $\varepsilon_{r}=2.2$ ) with thickness $h=1.6 \mathrm{~mm}$. The width of the microstrip is $w=2 \mathrm{~mm}$, and the length of a unit cell is $p=12 \mathrm{~mm}$. In our designed structure, the effective HMM is produced by loading lumped series capacitors $C=1 \mathrm{pF}$ in the $x$ direction. Lumped resistors $R=85 \Omega$ are loaded on the boundary of the sample to provide perfect matching conditions and avoid reflection from the sample boundary. The Huygens metasource experimental scheme is similar to the theoretical design in Fig. 2. Specifically, three voltage sources are used to construct an all-electric Huygens metasource in the center of the structure. The external integrated electric circuits and extra TL systems are exploited to modulate the phase delays of the constituent dipoles. ${ }^{33,49}$ These are marked by the red dots in Fig. 4(b). For clarity, we magnify the lumped capacitor elements in the inset of Fig. 4(b). The effective circuit models of the HMM are also shown at the bottom of the inset. The structural factor of a TL in a circuit-based HMM is defined as $g=Z_{0} / \eta_{\text {eff }}$, where $Z_{0}$ and $\eta_{\text {eff }}$ are the characteristic impedance and effective wave impedance of the TL, respectively. ${ }^{62,63}$ Especially, when $w>h, g=1 /[1.393+w / h+$ $(2 / 3) \ln (w / h+1.444)]$. The structural factor of our designed structure is $g \approx 0.3$. The metal used to design the microstrip is copper, and tin is plated on the surface of the metal to avoid oxidation. Especially, the copper in the microwave regime can be seen as the perfect electric conductor, and the loss of copper can be ignored. In fact, for the TL-based effective HMM, the loss mainly comes from the dielectric loss of the F4B substrate (the loss tangent is $\tan \delta=0.0079$ ), and it has been demonstrated by previous literature that the loss of the dielectric substrate has little effect on the bulk modes of circuit-based HMMs. ${ }^{6,14,20}$ Because the unit size in the TL system is much smaller than the wavelength, the effective permittivity of a $2 \mathrm{D}$ TL in a quasi-static TE-polarized solution can be written as (see more details in the Supplementary Material) ${ }^{62,63}$

$\varepsilon=2 C_{0} \cdot g / \varepsilon_{0}, \quad \mu_{x}=\frac{L_{0}}{g \cdot \mu_{0}}, \mu_{z}=\frac{L_{0}}{g \cdot \mu_{0}}-\frac{1}{\omega^{2} \cdot C \cdot d \cdot g \cdot \mu_{0}}$,

where $\varepsilon_{0}$ and $\mu_{0}$ are the permittivity and permeability of the vacuum, respectively; $\omega$ is the angular frequency; and $C_{0}$ and $L_{0}$ denote the capacitance and inductance, respectively, of the TL per unit length. ${ }^{62,63}$ In Eq. (1), $\varepsilon=3.57$ (red dashed line), $\mu_{x}=1$ (green dot dashed line), and the dependence of $\mu_{z}$ on the frequency (solid blue line) are shown in Fig. 4(c). Specifically, $\mu_{z} \approx 0$ when the frequency is $2.36 \mathrm{GHz}$ (green dotted line). When the frequency is smaller than this critical value, $\mu_{z}$ is negative. The dispersion relation of a circuit-based metamaterial is described by $\frac{k_{x}^{2}}{\varepsilon \cdot \mu_{z}}+\frac{k_{z}^{2}}{\varepsilon \cdot \mu_{x}}=\left(\frac{\omega}{c}\right)^{2}$. We use Eq. (3) to derive the effective parameters of the TLs and produce the results in Fig. 4(d). At the reference frequency of $1.5 \mathrm{GHz}, \mu_{z}=-1.47$, $\mu_{x}=1$, and $\varepsilon=3.57$. Under these parameters, we plot the IFC of this HMM using the solid blue line in Fig. 4(d). The IFC is a general hyperbola, in which the two asymptotes are represented 


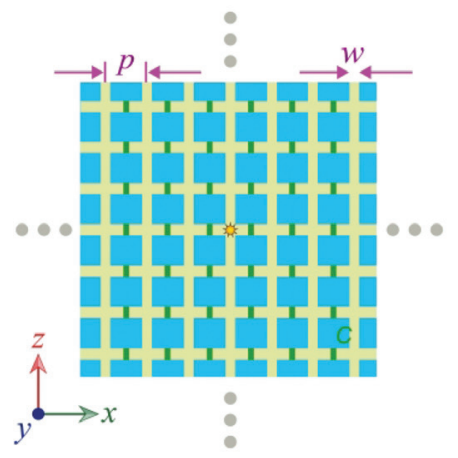

(a)

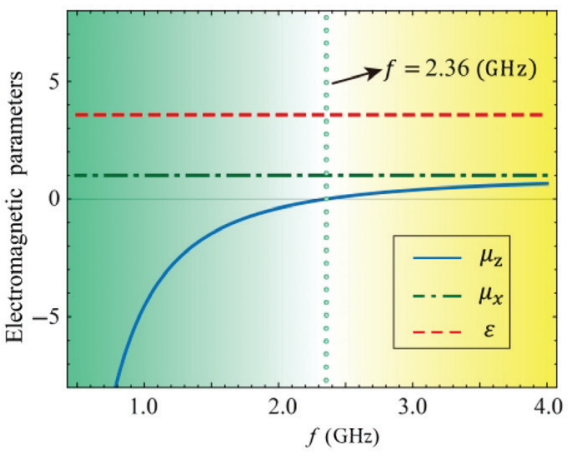

(c)

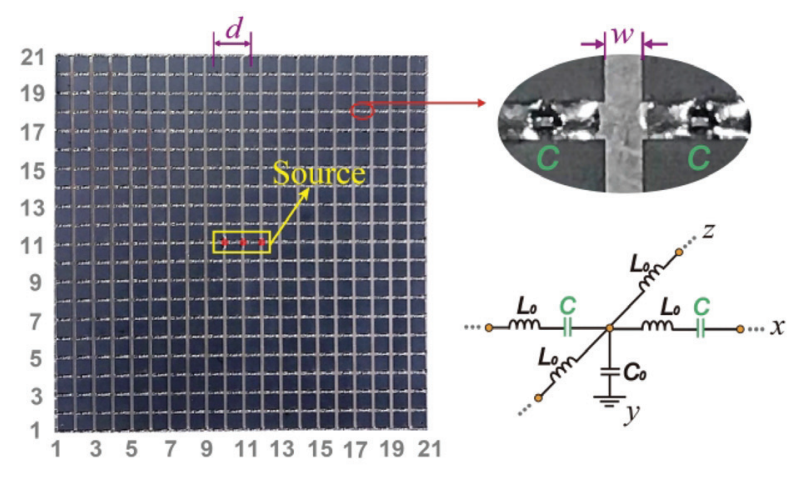

(b)

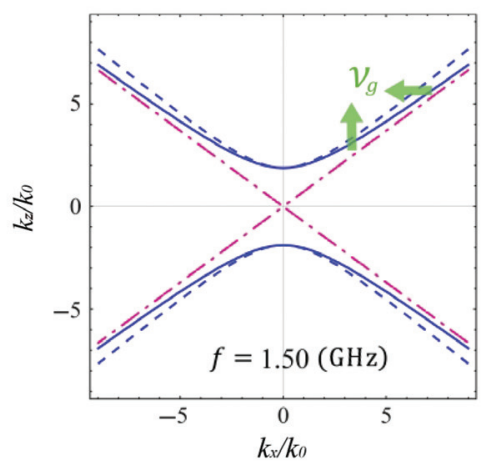

(d)

Fig. 4 (a) Schematic of a TL-based HMM structure with $p=12 \mathrm{~mm}, w=2 \mathrm{~mm}$, and $C=1 \mathrm{pF}$. (b) Prototype of a 2D TL with $21 \times 21$ unit cells and the related anisotropic $2 \mathrm{D}$-circuit model. The source is near the center of the sample. The inset shows the amplified lumped capacitors, which are loaded in the $x$ direction. (c) The effective anisotropic EM parameters are based on the TLs, where $\mu_{z}=0$ is marked using a green dotted line. (d) The IFC of a circuit-based HMM with $\mu_{z}=-1.47, \mu_{x}=1$, and $\varepsilon=3.57$ at $f=1.5 \mathrm{GHz}$. The asymptote is represented by purple dashed lines.

by red dashed lines. Because the DOS is largest along the directions of the two asymptotes, the energy inside the general HMM is mainly confined to two pathways determined by the directions of the asymptotes. ${ }^{23}$ The dashed blue line indicates that the IFC calculation frequency is slightly higher than that of the solid blue line. The green arrows indicate the direction of the energy flow. In Fig. 4(d), we see that the energy flow direction in an HMM can be controlled flexibly using the hyperbolic dispersion. In particular, the energy flow along the $z(x)$ direction is positive (negative). Therefore, hyperbolic bulk modes in HMMs can be used to achieve unidirectional excitation along various directions.

Full-wave simulations of the circuit-based system were performed using commercially available software (CST Microwave Studio) that included a finite-element frequency-domain solver. The HMMs are excited by a simple point source or effective all-electric Huygens metasources close to the center of the structure. For comparison, we consider a circuit-based isotropic medium with a closed IFC $\left(\varepsilon=3.57, \mu_{x}=1\right) .{ }^{20}$ The EM waves from a point source can propagate in all in-plane directions when the frequency is $1.5 \mathrm{GHz}$, as with the simulated distribution of an out-of-plane electric field $E_{y}$ in Fig. 5(a). Figures 5(b)-5(e) show the unidirectional excitation of a Huygens metasource in a normal material. We then simulate the radiation patterns of a point source and four types of Huygens metasources in circuit-based HMMs at a frequency of $1.5 \mathrm{GHz}$, as shown in Figs. 5(f)-5(j). The unidirectional excitation of hyperbolic bulk modes along the $z(x)$ direction is clearly the same (opposite) as that in a normal medium. This selective directional near-field coupling is enabled by the Huygens metasource composed of all-electric source components with a symmetry-associated inner freedom and promotes several symmetry-related near-field excitation behaviors. Especially, the Huygens metasource is strictly associated with the paritytime $(\hat{P} \hat{T}: \boldsymbol{r} \rightarrow-\boldsymbol{r}, t \rightarrow-t)$ symmetry and thus will inherit these symmetry properties naturally. ${ }^{49}$ In contrast, the spin dipoles and Janus dipoles are associated with the parity-reversal $(\hat{P}: \boldsymbol{r} \rightarrow-\boldsymbol{r})$ symmetry and time-reversal $(\hat{T}: t \rightarrow-t)$ symmetry, respectively. The metasources will be able to excite the mode pairs with the corresponding symmetry features. On the other hand, the Huygens metasource can be explained in terms of the time-averaged Poynting vector $\propto \operatorname{Re}\left[E^{*} \times H\right]$, which can produce fields associated with a net power flow in a given direction. ${ }^{48}$ Therefore, in addition to the near-field directionality, Huygens metasource can also be used to realize the far-field directionality, which is not possessed by spin and Janus dipoles. During the experimental process, signals are generated using a vector network analyzer (Agilent PNA Network Analyzer N5222A). One monopole source near the center of the sample is the point source used to excite the circuit-based prototype. 

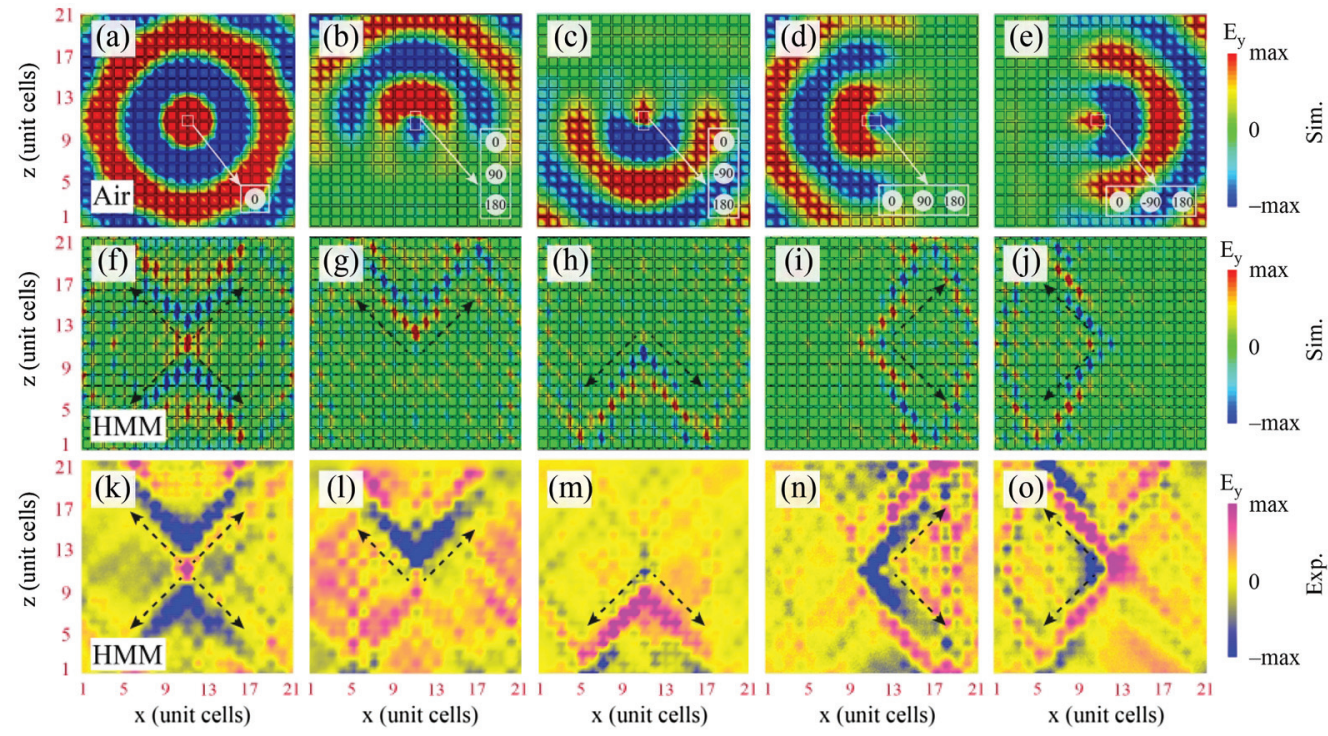

Fig. 5 (a) Simulated point dipole radiation patterns in the circuit-based normal medium. The EM waves can propagate along all directions. (b)-(e) Unidirectional propagation of the Huygens metasources in a circuit-based normal medium. Panels (f)-(j) correspond, respectively, to (a)-(e) but for the simulated radiation patterns in the circuit-based HMM. Panels (k)-(o) correspond, respectively, to $(\mathrm{a})-(\mathrm{e})$ but for the measured radiation patterns in the circuit-based HMM.

In addition, three vertical monopole sources at the subwavelength scale are used to construct the all-electric Huygens metasource. A small, 2-mm-long homemade rod antenna is employed to measure the out-of-plane electric field $E_{y}$ at a fixed height of $1 \mathrm{~mm}$ from the planar microstrip. The sample is placed on an automatic translation device with a scanning step of $1 \mathrm{~mm}$. This makes accurately probing the field distribution using a near-field scanning measurement feasible. The field amplitudes are normalized according to their respective maximum amplitudes. The experimentally measured results regarding the unidirectional excitation of hyperbolic bulk modes in Figs. 5(k)5(o) match the simulated results in Figs. 5(f) $-5(\mathrm{j})$ well. A comparison of the radiation patterns in the normal medium and the HMM shows that anomalous unidirectional excitation of hyperbolic bulk modes in the horizontal direction can be achieved using a Huygens metasource.

\section{Unidirectional Excitations of Hyperbolic Guided Modes Using a Spin Metasource}

Anomalous unidirectional excitation by an all-electric Huygens metasource in HMMs provides new ways to control EM waves within a near-field regime. Recently, special near-field dipoles, including spin dipoles ${ }^{64,65}$ Huygens dipoles ${ }^{66,67}$ Janus dipoles ${ }^{48}$ and composite spinning dipoles, ${ }^{68,69}$ have provided a good platform for studying interesting physical mechanisms such as transverse-spin-associated globally unique handedness ${ }^{70}$ bulk EM waves, ${ }^{35,71}$ bound states in continuum, ${ }^{72}$ and topological edge states. ${ }^{73,74}$ In this section, we demonstrate experimentally that a spin metasource can be used to produce the anomalous PSHE in a hyperbolic waveguide. Photons with different circular polarizations (optical spins) may propagate in different directions. This is referred to as the PSHE. ${ }^{23,33}$ Optical PSHE spin-orbit locking has attracted significant research attention and may be useful for many interesting fields of physics, such as chiral quantum optics ${ }^{64}$ and topological photonics ${ }^{75}$ within the near-field regime. In addition, the unidirectional excitation of optical modes is a fundamental prerequisite for numerous photonic applications, such as polarization beam splitters and directional radiation antennas. Here, we study abnormal directional excitation in a circuit-based hyperbolic waveguide. Because of their opendispersion IFCs, HMMs support propagation of high- $k$ modes with large effective refractive indices, thus allowing hyperbolic waveguides to be miniaturized. ${ }^{76,77}$ A near-field spin source couples with only one guided mode in the specific propagation direction determined by the handedness of the spin source. ${ }^{49}$

Here, the hyperbolic waveguide is composed of a core layer of HMM and two cladding layers made of a double positive (DPS) medium. As in the above section, the circuit-based HMM is produced using TLs by loading series lumped capacitors in the $x$ direction. Here, $C=5 \mathrm{pF}, w=2.8 \mathrm{~mm}$, and the other parameters remain unchanged. In this case, the structure factor is $g \approx 0.26$, and the effective EM parameters of the circuit-based HMM can be obtained using Eq. (3): $\mu_{z}=(1-1.316) \times$ $10^{18} / f^{2}, \mu_{x}=1$, and $\varepsilon=3.63$. In addition, a simple TL system without elements can produce a DPS medium $\left(\varepsilon_{D}=3.63\right.$ and $\mu_{D}=1$ ), as shown in Fig. 6(a). The corresponding effective circuit model of the unit structure of the circuit-based DPS medium is shown in Fig. 6(b). To emphasize the role of the HMM waveguide, we systematically compare a normal waveguide to a hyperbolic waveguide. The normal waveguide is composed of a DPS-medium core layer and two cladding layers of negative- $\mu$ (MNG) medium $\left[\varepsilon_{M}=3.63, \mu_{M}=(1-1.316) \times\right.$ $\left.10^{18} / f^{2}\right]^{33}$ The circuit-based MNG medium can be easily constructed using TLs by loading series lumped capacitors in both the $x$ and $z$ directions, as shown in Fig. 6(c). Analogous to Fig. 6(b), the effective circuit model of the circuit-based MNG medium is shown in Fig. 6(d). Based on the boundary conditions, the dispersion relation for the lowest-order guide modes for TE polarization in $\mathrm{HMM}^{78,79}$ and normal ${ }^{33}$ waveguides can be 


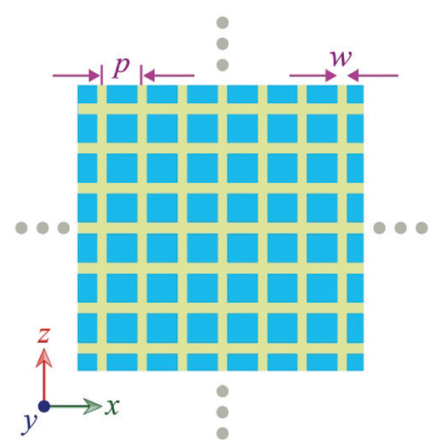

(a)

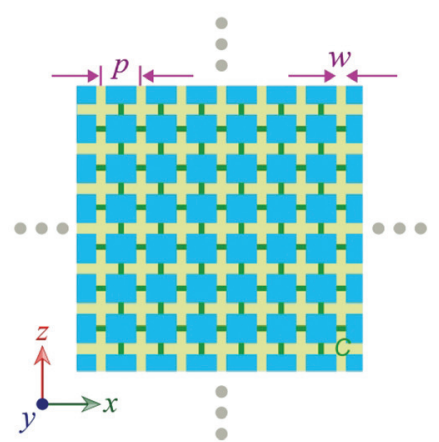

(c)

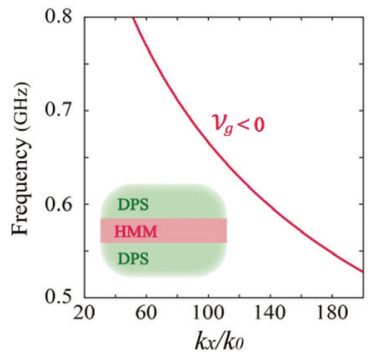

(e)

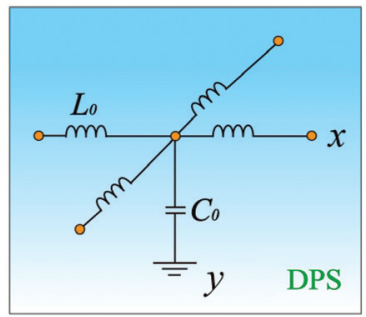

(b)

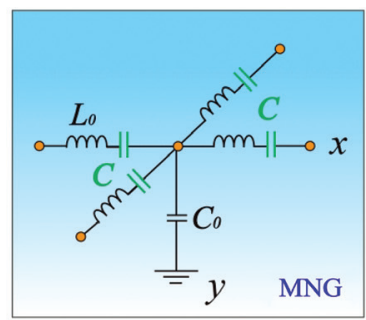

(d)

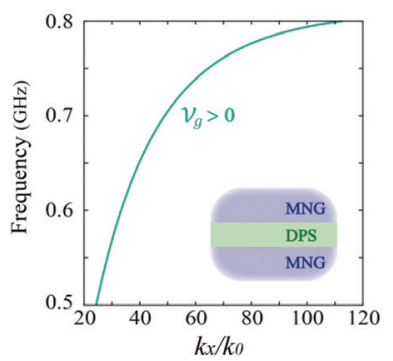

(f)
Fig. 6 (a), (b) Structure and related anisotropic 2D-circuit model of the TL-based DPS medium. Panels (c) and (d) are similar to (a) and (b) but for MNG media. Here, $p=12 \mathrm{~mm}, w=2.8 \mathrm{~mm}$, and $C=5 \mathrm{pF}$. (e) Dispersion relations of guided modes in a hyperbolic waveguide that is composed of a core HMM layer and two DPS-medium cladding layers. The structure is shown in the inset. Panel (f) is similar to (e), but for a normal waveguide, which is composed of a core layer of DPS medium and two MNG-medium cladding layers.

obtained (see more details in the Supplementary Material). Figures 6(e) and 6(f) show the dispersion relations of the circuitbased hyperbolic waveguide and normal waveguide, respectively, for a core width of $d_{c}$. The group velocity is calculated using $v_{g}=\partial \omega / \partial k_{x}$, which provides negative and positive group velocities for the hyperbolic and normal guided modes, respectively.

Figure 7(a) shows a schematic illustration of abnormal PSHE in a hyperbolic waveguide. The simulated electric-field distributions in Figs. 7(c) and 7(e) show that, for a counterclockwise (clockwise) near-field spin metasource, the hyperbolic guided mode along the interface runs from left (right) to right (left). For comparison, we also study directional excitation in a circuit-based normal waveguide [Fig. 7(b)]. The rightward

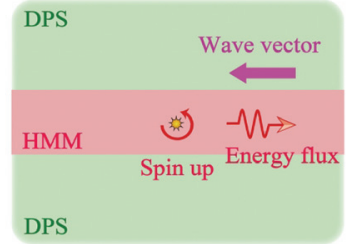

(a)

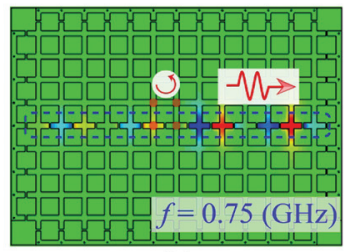

(c)

(e)

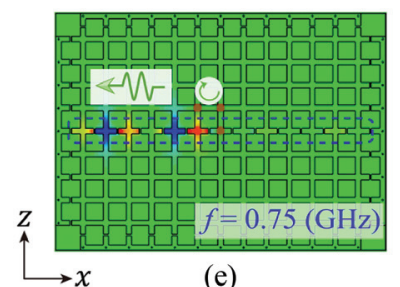

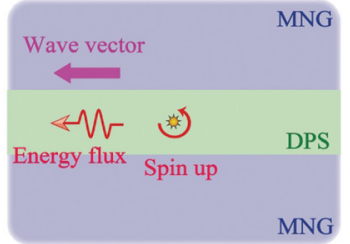

(b)

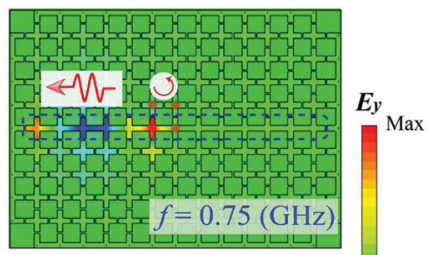

(d)

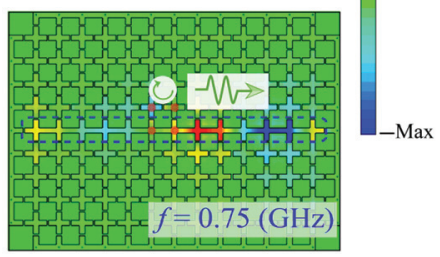

(f)
Fig. 7 Schematics of (a) anomalous PSHE in an HMM waveguide and (b) normal PSHE in a DPS waveguide. A source with specific handedness excites only a single-guided mode with a specific propagation direction. Anomalous unidirectional excitation occurs in the HMM waveguide. For a counterclockwise-spin metasource, only the guided modes that propagate from right to left and left to right are excited in the (c) HMM and (d) DPS waveguides, respectively. However, for a clockwise-spin metasource, only the guided modes that propagate from left to right and right to left are excited in the (e) HMM and (f) DPS waveguides, respectively.

(or leftward) unidirectional excitation of a counterclockwise (or clockwise) spin metasource in the hyperbolic waveguide changes to leftward (or rightward) unidirectional excitation in the normal waveguide, as shown in Figs. 7(d) and 7(f). Therefore, the direction of excitation in the hyperbolic waveguide is opposite that of normal PSHE. The guided mode with the directional wave vector in the PSHE comes from the spinmomentum locking mechanism. The propagation direction is determined by wave vector and energy flow parallelism or antiparallelism. In a normal waveguide, the direction of directional transmission is the same as that of the wave vector because the wave vector and energy flow are in the same direction. However, in a hyperbolic waveguide, the direction of transmission is reversed because the momentum and energy flow are in opposite directions. The extraordinary guided mode of HMMs makes them a good research platform for the study of abundant unidirectional transmission.

To conclude this section, experimental work that demonstrates anomalous unidirectional excitation of a circuit-based magnetic hyperbolic waveguide is discussed. Examples of circuit-based hyperbolic and normal waveguides are shown in Figs. 8(a) and 8(b), respectively. The core layers are marked by yellow dotted rectangles, and the all-electric spin metasources are marked using four dots. For a normal guided mode excited by a counterclockwise-spin metasource, the waves run along the 


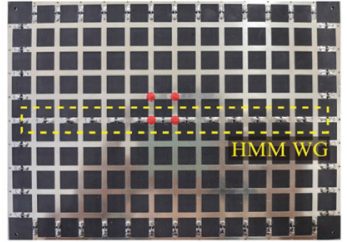

(a)

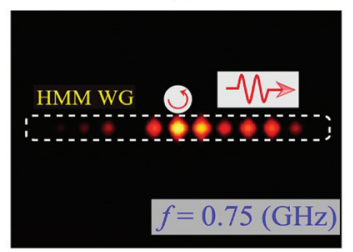

(c)

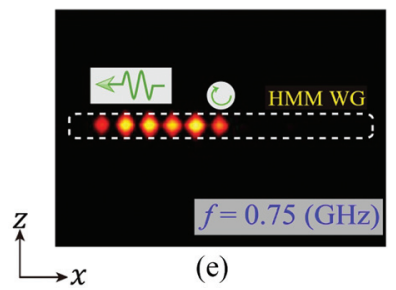

(e)

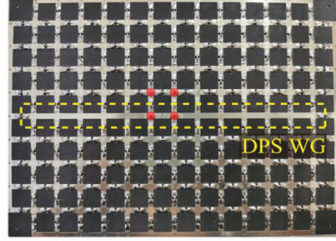

(b)

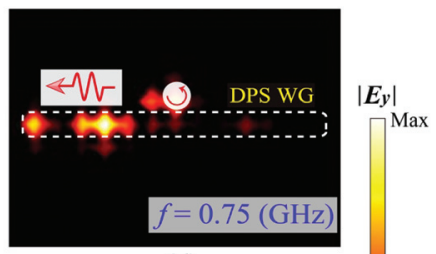

(d)

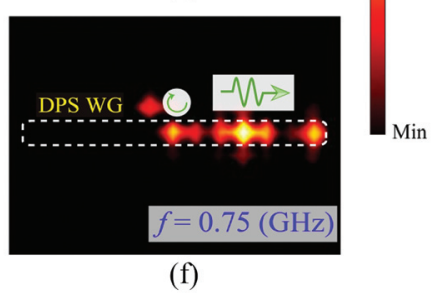

Fig. 8 (a) Experimental schematic of a circuit-based hyperbolic waveguide. Measured near-field distributions of $\left|E_{y}\right|$ for (c) counterclockwise and (e) clockwise spin metasources. Panels (b), (d), and (f) are similar to (a), (c), and (e), but for the circuit-based normal waveguide.

interface from right to left, as shown in Fig. 8(d). However, unidirectional transmission is reversed for the hyperbolic guided mode in Fig. 8(c). Similarly, for a normal guided mode excited by a clockwise spin metasource, the waves run along the interface from left to right in Fig. 8(f), whereas in a hyperbolic guided mode excited by a clockwise-spin metasource, the waves run along the interface from right to left in Fig. 8(e). Overall, the experimental field patterns in Fig. 8 are in good agreement with the simulated results in Fig. 7. Therefore, anomalous unidirectional excitation of hyperbolic guided modes is observed experimentally using an all-electric spin metasource.

\section{Conclusion}

Anomalous unidirectional excitation effects of hyperbolic modes were observed using near-field all-electric metasources. For the hyperbolic bulk mode, anomalous unidirectional excitation in the horizontal direction was achieved using a Huygens metasource. Moreover, using a hyperbolic waveguide, in which the group velocity and wave vector directions are opposite, anomalous unidirectional excitation of hyperbolic guided modes was demonstrated using a spin metasource. Based on the results from a hyperbolic bulk mode excited by a Huygens metasource and a hyperbolic guided mode excited by a spin metasource, we found that the circuit-based HMM is a good platform for the study of anomalous unidirectional excitation and has potential applications in near-field optical routing and energy transfer.

\section{Acknowledgments}

Z. W. Guo and Y. Long contributed equally to this work. This work was supported by the National Key R\&D Program of China
(Grant No. 2016YFA0301101); the National Natural Science Foundation of China (NSFC) (Grant Nos. 12004284, 11775159, 61621001, and 11935010); the Natural Science Foundation of Shanghai (Grant Nos. 18ZR1442800 and 18JC1410900); China Postdoctoral Science Foundation (Grant Nos. 2019TQ0232 and 2019M661605); the Shanghai Super Postdoctoral Incentive Program; and the Opening Project of Shanghai Key Laboratory of Special Artificial Microstructure Materials and Technology. The authors declare no conflicts of interest.

\section{References}

1. A. Poddubny et al., "Hyperbolic metamaterials," Nat. Photonics 7(12), 948-957 (2013).

2. P. Shekhar, J. Atkinson, and Z. Jacob, "Hyperbolic metamaterials: fundamentals and applications," Nano Converg. 1(1), 14 (2014).

3. L. Ferrari et al., "Hyperbolic metamaterials and their applications," Prog. Quantum Electron. 40, 1-40 (2015).

4. Z. W. Guo, H. T. Jiang, and H. Chen, "Hyperbolic metamaterials: from dispersion manipulation to applications," J. Appl. Phys. 127(7), 071101 (2020).

5. A. A. High et al., "Visible-frequency hyperbolic metasurface," Nature 522(7555), 192-196 (2015)

6. Z. W. Guo et al., "Focusing and super-resolution with partial cloaking based on linear-crossing metamaterials," Phys. Rev. Appl. 10(6), 064048 (2018).

7. X. Lin et al., "All-angle negative refraction of highly squeezed plasmon and phonon polaritons in graphene-boron nitride heterostructures," Proc. Natl. Acad. Sci. U. S. A. 114(26), 6717-6721 (2017).

8. J. Jiang, X. Lin, and B. L. Zhang, "Broadband negative refraction of highly squeezed hyperbolicpolaritons in 2D materials," Research 2018, 2532819 (2018).

9. K. Yu et al., "Loss-induced topological transition of dispersion in metamaterials," J. Appl. Phys. 119(20), 203102 (2016).

10. Z. W. Guo et al., "Actively controlling the topological transition of dispersion based on electrically controllable metamaterials," Appl. Sci. 8(4), 596 (2018).

11. P. X. Zheng et al., "Anomalous wave propagation in topological transition metasurfaces," Adv. Opt. Mater. 7(11), 1801483 (2019).

12. Z. W. Guo, H. T. Jiang, and H. Chen, "Linear-crossing metamaterials mimicked by multilayers with two kinds of single negative materials," J. Phys.: Photonics 2(1), 011001 (2020).

13. L. Shen et al., "Broadband enhancement of on-chip single-photon extraction via tilted hyperbolic metamaterials," Appl. Phys. Rev. 7(2), 021403 (2020).

14. Z. W. Guo, H. T. Jiang, and H. Chen, "Abnormal wave propagation in tilted linear-crossing metamaterials," Adv. Photonics Res. 2(1), 021403 (2020).

15. H. N. Krishnamoorthy et al., "Topological transitions in metamaterials," Science 336(6078), 205-209 (2012).

16. Z. Jacob, I. I. Smolyaninov, and E. E. Narimanov, "Broadband purcell effect: radiative decay engineering with metamaterials," Appl. Phys. Lett. 100(18), 181105 (2012).

17. Z. W. Liu et al., "Far-field optical hyperlens magnifying subdiffraction-limited objects," Science 315(5819), 1686 (2007).

18. I. I. Smolyaninov, Y.-J. Hung, and C. C. Davis, "Magnifying superlens in the visible frequency range," Science 315(5819), 1699-1701 (2007).

19. S.-A. Biehs, V. M. Menon, and G. S. Agarwal, "Long-range dipoledipole interaction and anomalous Förster energy transfer across a hyperbolic metamaterial," Phys. Rev. B 93(24), 245439 (2016).

20. Z. W. Guo et al., "Enhancement of electromagnetically induced transparency in metamaterials using long range coupling mediated by a hyperbolic material," Opt. Express 26(2), 627-641 (2018).

21. W. D. Newman et al., "Observation of long-range dipole-dipole interactions in hyperbolic metamaterials," Sci. Adv. 4(10), eaar5278 (2018). 
22. M. Neugebauer, P. Banzer, and S. Nechayev, "Emission of circularly polarized light by a linear dipole," $S c i$. $A d v .5(6)$, eaav7588 (2019).

23. P. V. Kapitanova et al., "Photonic spin Hall effect in hyperbolic metamaterials for polarization-controlled routing of subwavelength modes," Nat. Commun. 5(1), 3226 (2014).

24. K. Y. Bliokh et al., "Spin-orbit interactions of light," Nat. Photonics 9(12), 796-808 (2015).

25. K. Y. Bliokh, D. Smirnova, and F. Nori, "Quantum spin Hall effect of light," Science 348(6242), 1448-1451 (2015).

26. V. Mechelen and Z. Jacob, "Universal spin-momentum locking of evanescent waves," Optica 3(2), 118-126 (2016).

27. Y. Long, J. Ren, and H. Chen, "Intrinsic spin of elastic waves," Proc. Natl. Acad. Sci. U. S. A. 115(40), 9951-9955 (2018).

28. P. Shi et al., "Transverse spin dynamics in structured electromagnetic guided waves," Proc. Natl. Acad. Sci. U. S. A. 118(6), e2018816118 (2021).

29. Z. B. Zhang et al., "Controllable transport of nanoparticles along waveguides by spin-orbit coupling of light," Opt. Express 29(4), 6282-6292 (2021).

30. F. Feng et al., "On-chip plasmonic spin-Hall nanograting for simultaneously detecting phase and polarization singularities," Light-Sci. Appl. 9(1), 95 (2021).

31. J. S. Eismann et al., "Transverse spinning of unpolarized light," Nat. Photonics 15(2), 156-161 (2021).

32. J. Rodríguez-Fortuño et al., "Near-field interference for the unidirectional excitation of electromagnetic guided modes," Science 340(6130), 328-330 (2013).

33. Z. W. Guo et al., "Photonic spin Hall effect in waveguides composed of two types of single-negative metamaterials," Sci. Rep. 7(1), 7742 (2017).

34. F. Picardi et al., "Unidirectional evanescent-wave coupling from circularly polarized electric and magnetic dipoles: an angular spectrum approach," Phys. Rev. B 95(24), 245416 (2017).

35. L. Peng et al., "Transverse photon spin of bulk electromagnetic waves in bianisotropic media," Nat. Photonics 13(12), 878-882 (2019).

36. A. Nemilentsau et al., "Switchable and unidirectional plasmonic beacons in hyperbolic two-dimensional materials," Phys. Rev. B 99(20), 201405(R) (2019).

37. F. Q. Yang et al., "Actively controlled asymmetric edge states for directional wireless power transfer," Opt. Express 29(5), 7844-7857 (2021).

38. D. Marpaung, J. Yao, and J. Capmany, "Integrated microwave photonics," Nat. Photonics 13(2), 80-90 (2019).

39. M. Kerker, D.-S. Wang, and C. L. Giles, "Electromagnetic scattering by magnetic spheres," J. Opt. Soc. Am. 73(6), 765-767 (1983).

40. C. Pfeiffer and A. Grbic, "Metamaterial Huygens' surfaces: tailoring wave fronts with reflectionless sheets," Phys. Rev. Lett. 110(19), 197401 (2013).

41. A. Epstein, J. P. S. Wong, and G. V. Eleftheriades, "Cavity-excited Huygens' metasurface antennas for near-unity aperture illumination efficiency from arbitrarily large apertures," Nat. Commun. 7(1), 10360 (2016).

42. X. M. Zhang et al., "Dual-band unidirectional emission in a multilayered metal-dielectric nanoantenna," ACS Omega 2(3), 774-783 (2017).

43. M. Decker et al., "High-efficiency dielectric Huygens' surfaces," Adv. Opt. Mater. 3(6), 813-820 (2015).

44. S. Liu et al., "Huygens' metasurfaces enabled by magnetic dipole resonance tuning in split dielectric nanoresonators," Nano Lett. 17(7), 4297-4303 (2017).

45. S. Nechayev et al., "Huygens' dipole for polarization-controlled nanoscale light routing," Phys. Rev. A 99(4), 041801(R) (2019).

46. M. I. Abdelrahman et al., "Experimental demonstration of spectrally broadband Huygens sources using low-index spheres," $A P L$ Photonics 4(2), 020802 (2019).
47. K. Chen et al., "A reconfgurable active huygens' metalens," Adv. Mater. 29(17), 1606422 (2017).

48. F. Picardi, A. V. Zayats, and F. J. Rodríguez-Fortuño, "Janus and Huygens dipoles: near-field directionality beyond spin-momentum locking," Phys. Rev. Lett. 120(11), 117402 (2018).

49. Y. Long et al., "Designing all-electric subwavelength metasources for near-field photonic routings," Phys. Rev. Lett. 125(15), 157401 (2020).

50. S. J. Zeng et al., "Unidirectional excitation of plasmonic waves via a multilayered metal-dielectric-metal Huygens' nanoantenna," Opt. Lett. 43(13), 3053-3056 (2018).

51. X. Lin et al., "Chiral plasmons with twisted atomic bilayers," Phys. Rev. Lett. 125(7), 077401 (2020).

52. Y. H. Zhong et al., "Toggling near-field directionality via polarization control of surface waves," Laser Photonics Rev. 15(4), 2000388 (2021).

53. C. Shi et al., "Observation of acoustic spin," Natl. Sci. Rev. 6(4), 707-712 (2019).

54. Y. Long et al., "Symmetry selective directionality in near-field acoustics," Nat. Sci. Rev. 7(6), 1024-1035 (2020).

55. M. Wang et al., "Magnetic spin-orbit interaction of light," LightSci. Appl. 7(1), 14 (2018).

56. M. Neugebauer et al., "Magnetic and electric transverse spin density of spatially confined light," Phys. Rev. X 8(2), 021042 (2018).

57. S. S. Kruk et al., "Magnetic hyperbolic optical metamaterials," Nat. Commun. 7(1), 11329 (2016).

58. Y. H. Yang et al., "Magnetic hyperbolic metasurface: concept, design, and applications," Adv. Sci. 5(12), 1801495 (2018).

59. J. S. Gomez-Diaz and A. Alù, "Flatland optics with hyperbolic metasurfaces," ACS Photonics 3(12), 2211-2224 (2016).

60. Y. Mazor and A. Alù, "Nonreciprocal hyperbolic propagation over moving metasurfaces," Phys. Rev. B 99(4), 045407 (2019).

61. L. Novotny and B. Hecht, Principles of Nano-Optics, Cambridge University Press (2012).

62. Y. Q. Wang et al., "Circuit-based magnetic hyperbolic cavities," Phys. Rev. Appl. 13(4), 044024 (2020).

63. Y. Q. Chen et al., "Experimental demonstration of the magnetic field concentration effect in circuit-based magnetic near-zero index media," Opt. Express. 28(11), 17064-17075 (2020).

64. P. Lodahl et al., "Chiral quantum optics," Nature 541(7638), 473-480 (2017).

65. S. Nechayev and P. Banzer, "Mimicking chiral light-matter interaction," Phys. Rev. B 99(24), 241101(R) (2019).

66. M. Liu et al., "Huygens' metadevices for parametric waves," Phys. Rev. X 8(3), 031077 (2018).

67. A. Bag et al., "Transverse Kerker scattering for angstrom localization of nanoparticles," Phys. Rev. Lett. 121(19), 193902 (2018).

68. M. F. Picardi et al., "Experimental demonstration of linear and spinning Janus dipoles for polarisation- and wavelength-selective near-field coupling," Light-Sci. Appl. 8(1), 52 (2019).

69. J. E. Vázquez-Lozano, A. Martínez, and F. J. Rodríguez-Fortuño, "Near-field directionality beyond the dipole approximation: electric quadrupole and higher-order multipole angular spectra," Phys. Rev. Appl. 12(2), 024065 (2019).

70. X. Piao, S. Yu, and N. Park, "Design of transverse spinning of light with globally unique handedness," Phys. Rev. Lett. 120(20), 203901 (2018).

71. M. Kim et al., "Observation of enhanced optical spin Hall effect in a vertical hyperbolic metamaterial," ACS Photonics 6(10), 2530-2536 (2019).

72. G. Zito et al., "Observation of spin-polarized directive coupling of light at bound states in the continuum," Optica 6(10), 1305-1312 (2019).

73. Y. Li et al., "Topological LC-circuits based on microstrips and observation of electromagnetic modes with orbital angular momentum," Nat. Commun. 9(1), 4598 (2018).

74. T. Stauber et al., "Unidirectional plasmonic edge modes on general two-dimensional materials," 2D Mater. 6(4), 045023 (2019). 
75. T. Ozawa et al., "Topological photonics," Rev. Mod. Phys. 91(1), 015006 (2019).

76. W. J. Ji et al., "Theory and experimental observation of hyperbolic media based on structural dispersions," Phys. Rev. Mater. 4(10), 105202 (2020).

77. J. C. Fu et al., "Microwave waveguide-type hyperbolic metamaterials," Adv. Photonics Res. 2(2), 2000043 (2020).

78. Y. R. He, S. L. He, and X. D. Yang, "Optical field enhancement in nanoscale slot waveguides of hyperbolic metamaterials," Opt. Lett. 37(14), 2907-2909 (2012).

79. V. E. Babicheva et al., "Finite-width plasmonic waveguides with hyperbolic multilayer cladding," Opt. Express 23(8), 9681-9689 (2015).

Zhiwei Guo received his PhD in physics from Tongji University in 2019. Currently, he is a postdoctoral fellow in the School of Physics Science and Engineering at Tongji University. His current research interests include metamaterials, topological photonics, and non-Hermitian physics.

Yang Long received his $\mathrm{PhD}$ in physics from Tongji University in 2020. His current research interests include spin angular momentum in classical waves, topological physics and the applications of machine learning on physics.

Haitao Jiang received his $\mathrm{PhD}$ in physics from Tongji University in 2005. Currently, he is a professor in the School of Physics Science and Engineering at Tongji University. His current research interests include photonic crystals, topological photonics, and metamaterials.

Jie Ren received his $\mathrm{PhD}$ in condensed matter physics from National University of Singapore. Currently, he is a professor in the School of Physics Science and Engineering at Tongji University. His current research interests include quantum phononics, wave spin, topological metamaterials, and artificial intelligence.

Hong Chen received his $\mathrm{PhD}$ in condensed matter physics from Shanghai Jiaotong University in 1986. Currently, he is a distinguished professor in the School of Physics Science and Engineering at Tongji University. His recently research interests include photonic crystals, metamaterials, plasmonics, and artificial microstructures for manipulation of classical and quantum waves. 